\title{
Linear Conforming and Nonconforming Upwind Finite Elements for the Convection-Diffusion Equation
}

\author{
Ph. Caussignac and R. Touzani \\ Département de Mathématiques, Ecole Polytechnique Fédérale, CH 1015 \\ Lausanne, Switzerland
}

[Received 10 January 1986 and in revised form 24 June 1987]

\begin{abstract}
A conforming and a nonconforming method for the approximation of the stationary 2-D convection-diffusion equation at high Péclet number are presented. Convergence of order $\boldsymbol{h}$ is proved in a generic case and, for a particular choice of the upwind schemes, a discrete maximum principle is established under very unrestrictive conditions on the mesh. Results of numerical computations are produced to show the practical convergence of these methods.
\end{abstract}

\section{Introduction}

WE consider the following steady-state convection-diffusion equation for the scalar field $u$.

$$
-\kappa \Delta u+b \cdot \nabla u+c u=f
$$

in a 2-D domain $\Omega$ together with suitable boundary conditions; the external velocity field $b$, the source $f$, and the constants $k>0$ and $c \geqslant 0$ are given.

It is well known that centred finite-difference or standard finite-element approximations of the convective term in (1.1) lead to numerical instabilities or wiggles when $\boldsymbol{K}$ is small. Many upwind schemes were proposed to insure stability (see Richtmyer \& Morton (1967) and Thomasset (1981) and references therein). All kinds of methods are encountered; we believe that a good one must be stable and precise enough and easy to implement, and-if possible-its convergence and a discrete version of the maximum principle should be proved.

In this paper, we construct two upwind linear triangular finite-element methods that are easy to code. We prove, in a particular case, the monotonicity of the matrix of the linear system and convergence results for simplified schemes. The upwind process is very similar to those of Hughes et al. (1979) and Brooks \& Hughes (1982) for quadrilaterals. The monotonic scheme is obtained by moving quadrature points, and we should mention that-in the nonconforming case at least-the idea is due to Dervieux (see Thomasset, 1981). The local upwind coefficients are required to satisfy simple bounds in order to have the maximum principle. This is an advantage of our method over those requiring full upwinding, as for example that of Ohmori \& Ushijima (1984) or some of those described in Ikeda (1983).

In Section 2, we summarize the variational formulation of the continuous problem. The approximation with the conforming element and its upwind version are given in Section 3, and monotonicity is proved in Section 4. We proceed in 
the same way for the nonconforming element in Section 5 and Section 6 . Convergence results are established in Section 7, whereas Section 8 is devoted to numerical results and a conclusion.

The monotonicity results of Section 4 and Section 6 are valid for particular schemes and arbitrary non-negative values of the constant $c$ appearing in (1.1), provided that the mass matrix is (positive) diagonal. As far as the convergence estimates of Section 3 of Section 7 are concerned, we have to assume $c>0$; since the standard schemes converge for large $k$ or small mesh size $h$, our results are stated for values of $\kappa / h$ that are small beside the maximum of the velocity modulus.

\section{Summary of the continuous problem}

We assume, for simplicity, that the open bounded domain $\Omega \subset \mathbb{P}^{2}$ with boundary $\Gamma$ is polygonal. As usual, $\mathrm{L}^{2}(\Omega)$ will denote the space of squareintegrable functions on $\Omega$, equipped with the norm $\|\cdot\|_{0, \Omega}$, and $\mathrm{H}_{0}^{1}(\Omega)$ the Sobolev space of functions vanishing on $\Gamma$ which, together with their first-order derivatives, are in $\mathrm{L}^{2}(\Omega)$. The norm on $\mathrm{H}_{0}^{1}(\Omega)$ can be chosen as $|u|_{1, \Omega}=\|\nabla u\|_{0, \Omega}$; the velocity is assumed to have continuous first-order partial derivatives, that is, $b \in C^{1}(\bar{\Omega})^{2}$. Using the notation $\langle\bullet, \bullet\rangle$ for the inner product of $\mathrm{L}^{2}(\Omega)$ and the one of $\mathrm{L}^{2}(\Omega)^{2}$ as well, we define the bilinear form

$$
a(u, v)=\kappa\langle\nabla u, \nabla v\rangle+\langle b \cdot \nabla u, v\rangle+c\langle u, v\rangle, \quad \kappa>0, \quad c \geqslant 0 .
$$

A weak variational formulation of the problem corresponding to equation (1.1) with homogeneous Dirichlet boundary conditions reads:

$$
\text { Find } u \in \mathrm{H}_{0}^{1}(\Omega) \text { such that } a(u, v)=\langle f, v\rangle \forall v \in \mathrm{H}_{0}^{1}(\Omega) \text {. }
$$

Integrating equation (2.1) by parts yields the coercivity of $a(\bullet, \bullet)$, provided that $\operatorname{div} b$ is small enough. Without loss of generality, we assume from now on that

$$
\operatorname{div} b(x)=0 \quad(x \in \Omega),
$$

and shall make later some comments on the case where this condition is not met. Existence, uniqueness, and a weak form of the maximum principle are summarized in the following lemma.

LEMMA 2.1 For $f \in \mathrm{L}^{2}(\Omega)$, Problem (2.2) has one and only one solution $u$. Furthermore, if $f \geqslant 0$, one has $u \geqslant 0$.

Proof. Existence and uniqueness follow from the Lax-Milgram theorem. For the maximum principle, see Gilbarg \& Trudinger (1977: Thm 8.1).

Remark. The cases of inhomogeneous Dirichlet or mixed Dirichlet-Neumann boundary conditions can be reduced to the formulation (2.2) by putting the inhomogeneity in the right-hand side. Similarly, the pure Neumann problem can be solved, provided that a compatibility condition is added. 


\section{Linear conforming approximation}

Let $\tau_{h}$ be an admissible triangular mesh of $\bar{\Omega}$ ( $h$ denotes the largest side); recall that a family $\left\{\tau_{h}\right\}_{h}$ of admissible triangulations is regular if the smallest angle $\theta_{h}$ remains bounded from below, i.e. $\theta_{h} \geqslant \theta_{0}>0$, as $h$ goes to zero (see Temam, 1984: pp. 73-74). We define the sets

$$
\begin{aligned}
\mathscr{S}_{h} & =\left\{S \in \vec{\Omega}: S \text { is a vertex of a triangle } K \in \tau_{h}\right\}, \\
\mathscr{S}_{h}^{0} & =\left\{S \in \mathscr{S}_{h}: S \notin \Gamma\right\}, \\
\Sigma^{K} & =\mathscr{S}_{h} \cap K \quad\left(K \in \tau_{h}\right), \\
\mathcal{N}^{K}(S) & =\Sigma^{K} \backslash\{S\} \quad\left(K \in \tau_{h}, S \in \Sigma^{K}\right),
\end{aligned}
$$

and finite-dimensional vector spaces

$$
\begin{aligned}
V_{h} & =\left\{v \in \mathrm{C}^{0}(\bar{\Omega}): v\left\lceil K \text { is a linear polynomial } \forall K \in \tau_{h}\right\},\right. \\
V_{0 h} & =\left\{v \in V_{h}: v(S)=0 \forall S \in \mathscr{S}_{h} \cap \Gamma\right\} .
\end{aligned}
$$

The standard $P_{1}$-approximation of Problem (2.2) is then given by:

Find $u_{h} \in V_{0 h}$ such that $a\left(u_{h}, v_{h}\right)=\left\langle f, v_{h}\right\rangle \forall v_{h} \in V_{0 h}$.

Since the space $V_{0 h} \subset \mathrm{H}_{0}^{1}(\Omega)$ is complete, we have the following result.

Lemma 3.1 If $f \in \mathrm{L}^{2}(\Omega)$, then Problem (3.7) has one and only one solution $u_{h}$.

At very low values of the diffusion coefficient $\kappa$, stability and convergence results for $u_{h}$ in the $\mathrm{H}_{0}^{1}$ norm are not interesting, since the constants in the estimates are proportional to $\kappa^{-1}$. Uniform estimates can be obtained in the norm

$$
\|u\|_{\kappa}=\left(\kappa|u|_{1, \Omega}^{2}+c\|u\|_{0, \Omega}^{2}\right)^{\frac{1}{2}}, \quad c>0,
$$

as quoted in the following lemma.

LEMMA 3.2 We assume that the solution $u$ of $(2.2)$ is in $\mathrm{H}^{2}(\Omega)$. Then, for $c>0$ and $\kappa \leqslant \kappa_{0}<\infty$, there exist positive constants $C_{1}$ and $C_{2}$, independent of $h$ and $\kappa$, such that

$$
\begin{aligned}
\left\|u_{h}\right\|_{\kappa} & \leqslant C_{1}\|f\|_{0, \Omega}, \\
\left\|u-u_{h}\right\|_{\kappa} & \leqslant C_{2} h|u|_{2, \Omega},
\end{aligned}
$$

where $\left.\right|^{\circ, \Omega}$ is the usual seminorm of the Sobolev space $\mathrm{H}^{2}(\Omega)$.

Proof. Noticing that $a(u, u)=\|u\|_{k}^{2}$, we see that the bound (3.9) follows directly from (3.7) and the Cauchy-Schwarz inequality. The error estimate (3.10) is obtained by a standard reasoning (see for example Ciarlet, 1978: Thm 3.2.2) combined with:

$$
|a(u, v)| \leqslant C\|u\|_{\kappa}|v|_{1, \Omega} .
$$

Let us now introduce the general form of the upwind approximation. First, we decompose the velocity at each vertex as

$$
\boldsymbol{b}(S)=\sum_{S^{\prime} \in \mathcal{N}^{K}(S)} B_{S S^{\prime}}^{K} \overline{S S^{\prime}}\left(S \in \mathscr{P}_{h}, K \in \tau_{h}\right)
$$


To obtain the upwind scheme, we replace the convective term in (2.1) by

$$
\begin{aligned}
b_{h}(u, v)= & \sum_{K \in \tau_{h}} \frac{|K|}{3} \sum_{S \in \Sigma^{K}}\left(\frac{1}{2}[v(S) b(S) \cdot \nabla u\lceil K-u(S) b(S) \cdot \nabla v\lceil K]\right. \\
& +\sum_{S^{\prime} \in \mathcal{N}^{K}(S)} \alpha_{S S^{\prime}}^{K} B_{S S^{\prime}}^{K}\left(\sigma _ { S S ^ { \prime } } ^ { K } \cdot \nabla u \lceil K ) \left(\sigma_{S S^{\prime}}^{K} \cdot \nabla v\lceil K) \quad\left(u, v \in V_{h}\right),\right.\right.
\end{aligned}
$$

where $|K|$ is the area of the triangle $K$. The local upwind parameters $\alpha_{S S^{\prime}}^{K}$ are such that

$$
\alpha_{S S^{\prime}}^{K} B_{S S^{\prime}}^{K} \geqslant 0 \text { and }\left|\alpha_{S S^{\prime}}^{K}\right| \leqslant \frac{1}{2} \quad\left(K \in \tau_{h} ; S, S^{\prime} \in \Sigma^{K}\right),
$$

and the upwind vectors $\sigma_{S S^{\prime}}^{K}$ satisfy

$$
\left|\boldsymbol{\sigma}_{s S^{\prime}}^{K}\right| \leqslant C h \quad\left(K \in \tau_{h} ; S, S^{\prime} \in \Sigma^{K}\right),
$$

where $C>0$ is independent of $K, S, S^{\prime}$, and $\kappa$. By defining

$$
a_{h}(u, v)=\kappa\langle\nabla u, \nabla v\rangle+b_{h}(u, v)+c\langle u, v\rangle \quad(\kappa>0, c \geqslant 0),
$$

the upwind approximation of Problem (2.2) reads

$$
\text { Find } u_{h} \in V_{0 h} \text { such that } a_{h}\left(u_{h}, v_{h}\right)=\left\langle f, v_{h}\right\rangle \forall v_{h} \in V_{0 h} \text {. }
$$

The coercivity of $a_{h}(\bullet, \bullet)$ implies the existence and uniqueness of the solution $u_{h}$ of this problem.

ExAmples. If we set

$$
\sigma_{S S^{\prime}}^{K}=\overline{S S^{\prime}} \quad\left(K \in \tau_{h} ; S, S^{\prime} \in \Sigma^{K}\right),
$$

and use the linearity of the functions in $V_{h}$, the formula (3.12) can be written as

$$
\begin{array}{r}
b_{h}\left(u_{h}, v_{h}\right)=\sum_{K \in \tau_{h}} \frac{|K|}{6}\left(\sum _ { S \in \Sigma ^ { K } } \sum _ { S ^ { \prime } \in \mathcal { N } ^ { K } ( S ) } \left[v_{h}\left(S+\alpha_{S S^{\prime}}^{K}\left(S^{\prime}-S\right)\right) B_{S S^{\prime}}^{K} \overline{S S^{\prime}} \cdot \nabla u_{h} \uparrow K\right.\right. \\
\left.-u_{h}\left(S-\alpha_{S S^{\prime}}^{K}\left(S^{\prime}-S\right)\right) B_{S S^{\prime}}^{K} \overline{S S^{\prime}} \cdot \nabla v_{h}\lceil K]\right),
\end{array}
$$

which shows that the standard numerical integration of the convective term with quadrature points at $S \in \Sigma^{K}$ is replaced by a formula with 'upwind' points at $S \pm \alpha_{S S^{\prime}}^{K}\left(S^{\prime}-S\right)$. This scheme is analogous to that of Hughes et al. (1979) for quadrilaterals in which only one displaced quadrature point is used. The next schemes correspond to that of Brooks \& Hughes (1982), which is obtained by an argument based on the concept of artificial diffusivity. The first one is defined by

or

$$
\boldsymbol{\sigma}_{S S^{\prime}}^{K}=\boldsymbol{b}(S)\left|\overline{S S^{\prime}}\right| /|\boldsymbol{b}(S)| \quad\left(K \in \tau_{h} ; S, S^{\prime} \in \Sigma^{K}\right)
$$

$$
\boldsymbol{\sigma}_{S S^{\prime}}^{K}=\boldsymbol{b}(S) h /|\boldsymbol{b}(s)| \quad\left(K \in \tau_{h} ; S, S^{\prime} \in \Sigma^{K}\right),
$$

and the second one by

or

$$
\boldsymbol{\sigma}_{S S^{\prime}}^{K}=b^{K}\left|\overline{S S^{\prime}}\right| /\left|b^{K}\right| \quad\left(K \in \tau_{h} ; S, S^{\prime} \in \Sigma^{K}\right)
$$

$$
\sigma_{S S^{\prime}}^{K}=b^{K} h /\left|b^{K}\right| \quad\left(K \in \tau_{h} ; S, S^{\prime} \in \Sigma^{K}\right),
$$


where $\mathbf{b}^{K}$ is a mean value over $K$, for example:

$$
b^{K}=\frac{1}{3} \sum_{S \in \Sigma^{K}} b(S)
$$

The advantage of the schemes $(3.18)$ to $\left(3.19^{\prime}\right)$ is their lack of diffusivity in the direction perpendicular to the velocity or mean velocity.

\section{Maximum principle for the scheme (3.17)}

We denote the Lagrangian basis of $V_{h}$ by $\left\{\phi_{S}\right\}_{S \in \mathscr{S}_{h}}$ and introduce the operator $\boldsymbol{R}: \mathbb{R}^{2} \rightarrow \mathbb{R}^{2}$ of rotation by angle $-\frac{1}{2} \pi:$

$$
\boldsymbol{R}\left(x_{1}, x_{2}\right)=\left(x_{2},-x_{1}\right)
$$

A triangulation will be said to be of an acute type if no angle greater than $\frac{1}{2} \pi$ occurs. We set $N=\operatorname{dim} V_{h}$ and $N_{0}=\operatorname{dim} V_{0 h}$, and shall sometimes use a numbering of the vertices such that

$$
\mathscr{S}_{h}^{0}=\left\{S_{1}, S_{2}, \ldots, S_{N_{0}}\right\} .
$$

In order to prove properties of the matrix $\boldsymbol{A}$, with entries

$$
A_{i j}=a_{h}\left(\phi_{S_{j}}, \phi_{S_{i}}\right) \quad(1 \leqslant i, j \leqslant N),
$$

which lead to the maximum principle, some restrictions on the upwind parameters are necessary.

Hypothesis 4.1 $\forall K \in \tau_{h}, \forall S \in \Sigma^{K}, \forall S^{\prime}, S^{\prime \prime} \in \mathcal{N}^{K}(S)$, and if $B_{S S^{\prime}}^{K} \neq 0$ :

$$
2\left|\alpha_{S S^{\prime}}^{K}\right| \geqslant 1+\frac{3 K}{\left|B_{S S^{\prime}}^{K}\right|} \frac{\boldsymbol{R}\left(\overline{S^{\prime} S^{\prime \prime}}\right) \cdot \boldsymbol{R}\left(\overline{S^{\prime \prime} S}\right)}{4|K|^{2}}
$$

Remark. If the mesh is made of equilateral triangles, then the condition (4.3) reads

$$
2\left|\alpha_{S S^{\prime}}^{K}\right| \geqslant 1-1 / P_{S S^{\prime}}^{K}, \quad P_{S S^{\prime}}^{K}>0,
$$

where the local Péclet numbers are given by

$$
P_{S S^{\prime}}^{K}=\frac{1}{2}\left|B_{S S^{\prime}}^{K}\right| h^{2} / K \text {. }
$$

(The usual mesh Péclet number is defined as $\mathrm{Pe}=|\boldsymbol{b}| \boldsymbol{h} / \boldsymbol{K}$.)

We are ready to establish the properties of the matrix $\boldsymbol{A}$, in the case $c=0$.

LEMMA 4.2 Assuming that Hypothesis 4.1 holds and that the triangulation is of an acute type, one has, for the scheme (3.17) and $c=0$ :

$$
\begin{aligned}
\sum_{j=1}^{N} A_{i j}=0 & (1 \leqslant i \leqslant N), \\
A_{i i}>0 & (1 \leqslant i \leqslant N), \\
A_{i j} \leqslant 0 & (1 \leqslant i \neq j \leqslant N) .
\end{aligned}
$$


Proof. (1) It is easily seen that each term contributing to $A_{i j}$ contains $\nabla \phi_{s_{j}}$. The assertion then follows since $\sum_{j=1}^{N} \phi_{S_{j}}=1$.

(2) This is a consequence of the coercivity of $a_{h}(\bullet, \bullet)$.

(3) On the triangle $K$ whose vertices are $S, S^{\prime}, S^{\prime \prime}$, with positive oriented boundary, we have

$$
\nabla \phi_{S} \mid K=-\frac{1}{2|K|} R\left(\overline{\left(S^{\prime} S^{\prime \prime}\right.}\right)
$$

by using (3.11), (3.12), (3.15), (3.17), and the linearity of the basis functions, the contribution of $K$ to $a_{h}\left(\phi_{s^{\prime}}, \phi_{s}\right)$ can be written as

$$
\begin{aligned}
a_{1}^{K}+a_{2}^{K}= & \left(\frac{K}{8|K|} \boldsymbol{R}\left(\overline{S^{\prime} S^{\prime \prime}}\right) \cdot \boldsymbol{R}\left(\overline{S^{\prime \prime} S}\right)+\frac{|K|}{6} B_{S S^{\prime}}^{K}\left(1-2 \alpha_{S S^{\prime}}^{K}\right)\right) \\
& +\left(\frac{K}{8|K|} \boldsymbol{R}\left(\overline{S S^{\prime \prime}}\right) \cdot \boldsymbol{R}\left(\overline{S^{\prime \prime} S^{\prime}}\right)-\frac{|K|}{6} B_{S^{\prime} S}^{K}\left(1+2 \alpha_{S^{\prime} S}^{K}\right)\right) .
\end{aligned}
$$

Let us show that $a_{1}^{K}$, the first expression in large parentheses, is $\leqslant 0$. If $B_{S S^{\prime}}^{K} \leqslant 0$, then $B_{S S^{\prime}}^{K}\left(1-2 \alpha_{S S^{\prime}}^{K}\right) \leqslant 0$ since $B_{S S^{\prime}}^{K} \alpha_{S S^{\prime}}^{K} \geqslant 0$, and $a_{1}^{K} \leqslant 0$ because the triangulation is of acute type. Otherwise, if $B_{S^{\prime}}^{K}>0$, then $a_{1}^{K} \leqslant 0$ by inequality (4.3). A similar reasoning allows to show that $a_{2}^{K} \leqslant 0$.

Let us recall that a matrix is monotonic if its inverse exists and is positive. The next proposition states that the matrix $\overline{\boldsymbol{A}}$ of the linear system, with entries

$$
\bar{A}_{i j}=a_{h}\left(\phi_{s_{i}}, \phi_{S_{i}}\right) \quad\left(1 \leqslant i, j \leqslant N_{0}\right) \text {, }
$$

is monotonic.

Proposition 4.3 Assume that the triangulation is of an acute type, Hypothesis 4.1 holds, and the mass matrix is diagonal with positive diagonal entries. If furthermore, there exists an element $K \in \tau_{h}$ and vertices $S \in \Sigma^{K} \cap \Gamma$ and $S^{\prime} \in$ $\mathcal{N}^{K}(S) \cap \mathscr{S}_{h}^{0}$ such that either

$$
B_{S S^{\prime}}^{K}=0 \text { and the angle of } K \text { at } S \text { is less than } \frac{1}{2} \pi
$$

or

$$
B_{S^{\prime} S}^{K}>0
$$

then the matrix $\bar{A}$ for the scheme (3.17) is monotonic.

Proof. By Lemma 4.2, we have

$$
\begin{aligned}
\sum_{j=1}^{N_{0}} \bar{A}_{i j} \geqslant 0 & \left(1 \leqslant i \leqslant N_{0}\right), \\
\bar{A}_{i i}>0 & \left(1 \leqslant i \leqslant N_{0}\right), \\
\bar{A}_{i j} \leqslant 0 & \left(1 \leqslant i \neq j \leqslant N_{0}\right) .
\end{aligned}
$$

Now, in order to prove that $\overline{\boldsymbol{A}}$ is monotonic, it is sufficient (Berman \& Plemmons, 1979: Theorem 2.3) to show that $\sum_{j=1}^{N_{0}} \bar{A}_{i_{0} j}>0$, for some $i_{0} \in\left\{1,2, \ldots, N_{0}\right\}$. It follows from the proof of Lemma 4.2 that, if either condition (a) or (b) is 
satisfied, then $a_{h}\left(\phi_{S}, \phi_{S^{\prime}}\right)<0$. Hence, there exists an entry of $\boldsymbol{A}$, not belonging to $\bar{A}$, which is negative. Since $\sum_{S^{\prime} \in \mathscr{S}_{h}} a_{h}\left(\phi_{S^{\prime}}, \phi_{s}\right)=0$, we have the desired property.

The condition on the mass matrix occurring in the hypotheses of Proposition 4.3 can be achieved by numerical integration with quadrature points at the vertices. We now state the maximum principle and a stability property.

Corollary 4.4 Assume that the hypotheses of Proposition 4.3 hold, $f \in \mathrm{L}^{2}(\Omega)$, and the mass matrix is diagonal with positive diagonal entries if $c>0$. Then, Problem (3.15) with the scheme (3.17) has one and only one solution $u_{h}$ such that $u_{h} \geqslant 0$ if $f \geqslant 0$. Furthermore, if $c>0$ and $f \in \mathrm{L}^{\infty}(\Omega)$, there exists a constant $C>0$, independent of $h$ and $\kappa$, such that $\left\|u_{h}\right\|_{\infty} \leqslant C\|f\|_{\infty}$, where $\|\cdot\|_{\infty}$ is the usual norm of $\mathrm{L}^{\infty}(\Omega)$.

Proof. The first part of the assertion is a direct consequence of Proposition 4.3. To prove the stability property, it suffices to show that the induced $\ell^{\infty}$ norm of $\overline{\boldsymbol{A}}^{-1}$ is independent of $\kappa$. For $c>0$, we have $\overline{\boldsymbol{A}}=\tilde{\boldsymbol{A}}+\boldsymbol{M}$, with, $\forall i \in$ $\left\{1,2, \ldots, N_{0}\right\}$ :

$$
\sum_{j=1}^{N_{0}} \tilde{A}_{i j} \geqslant 0, \quad M_{i j}=m_{i} \delta_{i j} \quad\left(1 \leqslant j \leqslant N_{0}\right), \quad m_{i}>0 .
$$

The vector $z \in \mathbb{R}^{N_{0}}$ with components $z_{j}=\left(\min _{i} m_{i}\right)^{-1}\left(1 \leqslant j \leqslant N_{0}\right)$ satisfies $\sum_{j=1}^{N_{0}} \bar{A}_{i j} z_{j} \geqslant 1 \quad\left(1 \leqslant i \leqslant N_{0}\right)$; therefore $\left\|\bar{A}^{-1}\right\|_{\infty}=\|z\|_{\infty}=C$.

Remark. If $\operatorname{div} b \neq 0$, one has to replace (3.12) and (3.13) by

and

$$
\begin{aligned}
b_{h}(u, v)= & \sum_{K \in \tau_{h}} \frac{|K|}{3} \sum_{S \in \Sigma^{K}}(v(s) b(s) \cdot \nabla u\lceil K \\
& +\sum_{S^{\prime} \in \mathcal{N}^{\mathcal{K}}(S)} \alpha_{S S^{\prime}}^{K} B_{S S^{\prime}}^{K}\left(\boldsymbol{\sigma}_{S S^{\prime}}^{K} \cdot \nabla u\lceil K)\left(\boldsymbol{\sigma}_{S S^{\prime}}^{K} \cdot \nabla v\lceil K)\right),\right.
\end{aligned}
$$

$$
0 \leqslant \alpha_{S S^{\prime}}^{K} \leqslant 1, \quad \alpha_{S S^{\prime}}^{K}=0 \quad \text { if } B_{S S^{\prime}}^{K} \leqslant 0 .
$$

The same techniques allow us to show the validity of Corollary 4.4 in this case, provided that the condition (4.3) is replaced by

$$
\alpha_{S S^{\prime}}^{K} \geqslant 1+\frac{3 K}{B_{S S^{\prime}}^{K}} \frac{\boldsymbol{R}\left(S^{\prime} S^{\prime \prime}\right) \cdot \boldsymbol{R}\left(S^{\prime \prime} S\right)}{4|K|^{2}},
$$

if $B_{S S^{\prime}}^{K}>0$. For a mesh made of equilateral triangles, (4.7) reads

with

$$
\alpha_{S S^{\prime}}^{K} \geqslant 1-1 / P_{S S^{\prime}}^{K}, \quad P_{S S^{\prime}}^{K}>0,
$$

$$
P_{S s^{\prime}}^{K}=\frac{1}{2} B_{S s^{\prime}}^{K} \cdot h^{2} / K
$$

The case of equality corresponds to the recommended choice of Hughes et al. (1979). On the other hand, one can show that the standard approximation leads to a monotonic matrix if $P_{s s^{\prime}}^{K}<1$. Hence, a natural choice of the upwind 
parameters is, in this case,

$$
\alpha_{S s^{\prime}}^{K}= \begin{cases}0 & \text { if } P_{S s^{\prime}}^{K}<1 \\ 1-1 / P_{S S^{\prime}}^{K} & \text { otherwise }\end{cases}
$$

\section{The linear nonconforming approximation}

The nonconforming element presented here can be easily generalized to the ' $P_{1}-P_{0}$ ' finite element for solving Navier-Stokes equations (Thomasset, 1981). We consider again an admissible triangulation $\tau_{h}$ of $\bar{\Omega}$ and define the sets

$$
\begin{gathered}
\mathcal{M}_{h}=\left\{M \in \bar{\Omega}: M \text { is the midpoint of a side of a triangle } K \in \tau_{h}\right\}, \\
\mathcal{M}_{h}^{0}=\left\{M \in \mathcal{M}_{h}: M \notin \Gamma\right\}, \\
M^{K}=\mathcal{M}_{h} \cap K \quad\left(K \in \tau_{h}\right), \\
\mathcal{N}^{\prime K}(M)=M^{K} \backslash\{M\}, \quad\left(M \in \mathcal{M}_{h}\right)
\end{gathered}
$$

and finite-dimensional vector spaces

$$
\begin{gathered}
V_{h}^{\prime}=\left\{v \in \mathrm{L}^{2}(\Omega): v\left\lceil K \text { is a linear polynomial } \forall K \in \tau_{h} ; v\right. \text { is }\right. \\
\text { continuous at each point } \left.M \in \mathcal{M}_{h}^{0}\right\}, \\
V_{0 h}^{\prime}=\left\{v \in V_{h}^{\prime}: v(M)=0 \forall M \in \mathcal{M}_{h} \cap \Gamma\right\} .
\end{gathered}
$$

Since the space $V_{0 h}^{\prime}$ is an external approximation of $\mathrm{H}_{0}^{1}(\Omega)$, we must define the bilinear form as follows.

$$
\begin{aligned}
\tilde{a}_{h}(u, v)= & \sum_{K \in \tau_{h}}\left[\kappa\langle\nabla u, \nabla v\rangle_{K}+\frac{1}{2}\left(\langle b \cdot \nabla u, v\rangle_{K}-\langle b \cdot \nabla v, u\rangle_{K}\right)\right]+c\langle u, v\rangle, \\
& \kappa>0, \quad c \geqslant 0,
\end{aligned}
$$

where $\langle\cdot, \cdot\rangle_{K}$ is the inner product of $\mathrm{L}^{2}(K)$ or $\mathrm{L}^{2}(K)^{2}$.

Let us introduce the seminorm

$$
\|v\|_{h}=\left(\sum_{K \in \tau_{h}}\langle\nabla v, \nabla v\rangle_{K}\right)^{\frac{1}{2}}
$$

and the norm

$$
\|v\|_{1, h}=\left(\sum_{K \in \tau_{h}}\left(\langle v, v\rangle_{K}+\langle\nabla v, \nabla v\rangle_{K}\right)\right)^{\frac{1}{2}} .
$$

The nonconforming approximation of Problem (2.2) at zero-divergence velocity then reads:

$$
\text { Find } \bar{u}_{h} \in V_{0 h}^{\prime} \text { such that } \bar{a}_{h}\left(\bar{u}_{h}, v_{h}\right)=\left\langle f, v_{h}\right\rangle \quad \forall v_{h} \in V_{0 h}^{\prime} \text {. }
$$

As in the continuous case, we have the following discrete Poincaré inequality.

LEMMA 5.1. If $\tau_{h}$ belongs to a regular family of triangulations, then there exists $a$ constant $\bar{C}(\Omega)>0$ such that

$$
\left\|v_{h}\right\|_{0, \Omega} \leqslant \bar{C}(\Omega)\left\|v_{h}\right\|_{h} \quad \forall v_{h} \in V_{0 h}^{\prime},
$$

and consequently the seminorm (5.8) and the norm (5.9) are equivalent on $V_{0 h}^{\prime}$. 
Proof. See Temam (1984: Propn 4.13).

It follows from (5.7) that the bilinear form $\tilde{a}_{h}(\bullet, \bullet)$ is coercive on $V_{0 h}^{\prime}$ and hence we have the following result.

LEMMA 5.2. If $f \in \mathrm{L}^{2}(\Omega)$, the problem (5.10) has one and only one solution $\tilde{u}_{h}$.

In the norm:

$$
\|u\|_{\kappa, h}=\left(\kappa\|u\|_{h}^{2}+c\|u\|_{0, \Omega}^{2}\right)^{\frac{1}{2}}, \quad \kappa>0, \quad c>0,
$$

the form $a_{h}^{\prime}(\bullet, \bullet)$ is also coercive, and the Cauchy-Schwarz inequality gives us the stability result

$$
\left\|\bar{u}_{h}\right\|_{\kappa, h} \leqslant C_{1}\|f\|_{0, \Omega}
$$

where $C_{1}>0$ is independent of $h$ and $\kappa$.

To set up the nonconforming upwind approximation, we first decompose the velocity at the mid-side nodes as follows:

$$
b(M)=\sum_{M^{\prime} \in \mathcal{N}^{k}(M)} B_{M M^{\prime}}^{K} \cdot \overline{M M^{\prime}} \quad\left(M \in \mathcal{M}_{h}, K \in \tau_{h}\right),
$$

and require the upwind coefficients and vectors to satisfy

$$
\begin{gathered}
\alpha_{M M^{\prime}}^{K} B_{M M^{\prime}}^{K} \geqslant 0 \text { and }\left|\alpha_{M M^{\prime}}^{K}\right| \leqslant \frac{1}{2} \quad\left(K \in \tau_{h} ; M, M^{\prime} \in M^{K}\right), \\
\left|\sigma_{M M^{\prime}}^{K}\right| \leqslant C \mid \overline{M M^{\prime} \mid}\left(K \in \tau_{h} ; M, M^{\prime} \in M^{K}\right),
\end{gathered}
$$

where $C>0$ is independent of $K, M, M^{\prime}$, and $K$.

As in the conforming case, we introduce the upwind forms

$$
\begin{aligned}
b_{h}^{\prime}(u, v)= & \sum_{K \in \tau_{h}} \frac{|K|}{3} \sum_{M \in M^{K}}\left(\frac{1}{2}(v(M) b(M) \cdot \nabla u\lceil K-u(M) b(M) \cdot \nabla v\lceil K)\right. \\
& +\sum_{M^{\prime} \in \mathcal{N}^{\prime} K(M)} \alpha_{M M^{\prime}}^{K} B_{M M^{\prime}}^{K}\left(\sigma_{M M^{\prime}}^{K} \cdot \nabla u\lceil K)\left(\sigma_{M M^{\prime}}^{K} \cdot \nabla v\lceil K)\right) \quad\left(u, v \in V_{h}^{\prime}\right)\right. \\
a_{h}^{\prime}(u, v)= & \sum_{K \in \tau_{h}} K\langle\nabla u, \nabla v\rangle_{K}+b_{h}^{\prime}(u, v)+c\langle u, v\rangle, \quad K>0, \quad c \geqslant 0,
\end{aligned}
$$

and the upwind approximate problem reads:

$$
\text { Find } u_{h} \in V_{0 h}^{\prime} \text { such that } a_{h}^{\prime}\left(u_{h}, v_{h}\right)=\left\langle f, v_{h}\right\rangle \quad \forall v_{h} \in V_{0 h}^{\prime} \text {. }
$$

Obviously, this problem has a unique solution. The schemes analogous to (3.17) and (3.18) are

$$
\begin{aligned}
& \boldsymbol{\sigma}_{M M^{\prime}}^{K}=\overline{M M^{\prime}} \quad\left(K \in \tau_{h} ; M, M^{\prime} \in M^{K}\right), \\
& \boldsymbol{\sigma}_{M M^{\prime}}^{K}=b(M)\left|\overline{M M^{\prime}}\right| /|b(M)| \quad\left(K \in \tau_{h} ; M, M^{\prime} \in M^{K}\right),
\end{aligned}
$$

and we obtain in a similar way those analogues to $\left(3.18^{\prime}\right)-\left(3.19^{\prime}\right)$. 
6. Maximum principle for the scheme (5.20)

We set $N=\operatorname{dim} V_{h}^{\prime}$ and $N_{0}=\operatorname{dim} V_{0 h}^{\prime}$ and assume that the mid-side nodes are numbered so that $\mathcal{M}_{h}^{0}=\left\{M_{1}, M_{2}, \ldots, M_{N_{0}}\right\}$.

Let $\boldsymbol{A}^{\prime}$ be the matrix with entries

$$
A_{i j}^{\prime}=a_{h}^{\prime}\left(\phi_{M_{j}}, \phi_{M_{i}}\right) \text {, }
$$

where $\left\{\phi_{M_{i}}\right\}_{j=1}^{N}$ is the usual Lagrangian basis of $V_{h}^{\prime}$.

We make the following assumption.

Hypothesis 6.1 $\forall K \in \tau_{h}, \forall M \in M^{K}, \forall M^{\prime}, M^{\prime \prime} \in \mathcal{N}^{\prime K}(M)$, and if $B_{M M^{\prime}}^{K} \neq 0$ :

$$
2\left|\alpha_{M M^{\prime}}^{K}\right| \geqslant 1+\frac{12 \kappa}{\left|B_{M M^{\prime}}^{K}\right|} \frac{\boldsymbol{R}\left(\overline{M^{\prime \prime} M^{\prime}}\right) \cdot \boldsymbol{R}\left(\overline{M M^{\prime \prime}}\right)}{|K|^{2}},
$$

where $\boldsymbol{R}$ is defined by (4.1).

If the mesh is made of equilateral triangles, the last condition reads:

$$
2\left|\alpha_{M M^{\prime}}^{K}\right| \geqslant 1-1 / P_{M M^{\prime}}^{K}, \quad P_{M M^{\prime}}^{K}>0,
$$

where the local Péclet numbers are given by:

$$
P_{M M^{\prime}}^{K}=\frac{1}{8}\left|B_{M M^{\prime}}^{K}\right| h^{2} / K .
$$

One can copy the proof of Lemma 4.2 to obtain a similar lemma, leading to the monotonicity of the matrix $\overline{\boldsymbol{A}}^{\prime}$, with entries

$$
\bar{A}_{i j}^{\prime}=a_{h}^{\prime}\left(\phi_{M_{j}}, \phi_{M_{i}}\right) \quad\left(1 \leqslant i, j \leqslant N_{0}\right) .
$$

Note that, here, the exact mass matrix is diagonal with positive diagonal entries.

Proposition 6.2 Assume that the triangulation is of acute type, Hypothesis 6.1 holds, and there exist $K \in \tau_{h}, M \in M^{K} \cap \Gamma$, and $M^{\prime} \in \mathcal{N}^{\prime} K(M) \cap \mathcal{M}_{h}^{0}$ such that either

(a) $B_{M M^{\prime}}^{K}=0$ and the angle at the vertex opposite to $M^{\prime}$ in $K$ is less than $\frac{1}{2} \pi$ or

(b) $B_{M}^{K} M^{\prime}>0$.

Then the matrix $\bar{A}^{\prime}$ for the scheme (5.20) is monotonic and $\sum_{j=1}^{N_{0}} A_{i j}^{\prime} \geqslant 0(1 \leqslant i \leqslant$ $N_{0}$ ).

Proof. The proof is similar to that of Proposition (4.3); here, we have

$$
\nabla \phi_{M} \mid K=\frac{2}{|K|} R\left(\overline{M^{\prime \prime} M^{\prime}}\right)
$$

on triangle $K$ with mid-side nodes $M, M^{\prime}, M^{\prime \prime}$ and positive-oriented boundary.

The reasoning leading to the conclusion of Corollary 4.4 applies here too, and gives the maximum principle and stability.

Corollary 6.3 Assume that the hypotheses of Proposition 6.2 hold and $f \in \mathrm{L}^{2}(\Omega)$. Then, Problem (5.19) with the scheme (5.20) has one and only one solution $u_{h}$ which satisfies

$$
u_{h}\left(M_{i}\right) \geqslant 0 \quad\left(1 \leqslant i \leqslant N_{0}\right) \text { if } f \geqslant 0 .
$$


Furthermore, if $c>0$ and $f \in \mathrm{L}^{\infty}(\Omega)$, then there exists $C>0$, independent of $h$ and $K$, such that

$$
\max _{i \leqslant i \leqslant N_{0}}\left|u_{h}\left(M_{i}\right)\right| \leqslant C\|f\|_{\infty}
$$

Remark. So far, we have given the nonconforming scheme for zero-divergence velocity. If $\operatorname{div} b \not \equiv 0$, we replace (5.17) and (5.15) by

$$
\begin{aligned}
b_{h}^{\prime}(u, v) & =\sum_{K \in \tau_{h}} \frac{|K|}{3} \sum_{M \in M^{K}}(v(M) b(M) \cdot \nabla u\lceil K \\
& +\sum_{M^{\prime} \in \mathcal{N}^{\prime}(M)} \alpha_{M M^{\prime}}^{K} B_{M M^{\prime}}^{K}\left(\sigma_{M M^{\prime}}^{K} \cdot \nabla u\lceil K)\left(\sigma_{M M^{\prime}}^{K} \cdot \nabla v\lceil K)\right) \quad\left(u, v \in V_{h}^{\prime}\right),\right. \\
& 0 \leqslant \alpha_{M M^{\prime}}^{K} \leqslant 1, \quad \alpha_{M M^{\prime}}^{K}=0 \quad \text { if } B_{M M^{\prime}}^{K}=0 .
\end{aligned}
$$

The condition (6.2) becomes

$$
\alpha_{M M^{\prime}}^{K} \geqslant 1+\frac{12 \kappa}{B_{M M^{\prime}}^{K}} \frac{\boldsymbol{R}\left(\overline{M^{\prime \prime} M^{\prime}}\right) \cdot \boldsymbol{R}\left(\overline{M M^{\prime \prime}}\right)}{|K|^{2}}, \quad B_{M M^{\prime}}^{K}>0,
$$

and allows us to prove that the conclusion of Corollary 6.3 applies to this case too.

\section{Convergence results}

Throughout this section, we shall assume the solution $u$ of the continuous problem (2.2) to be in $\mathrm{H}^{2}(\Omega)$ and set

$$
B=\max _{x \in \bar{\Omega}}|\boldsymbol{b}(x)| .
$$

Our schemes belong to the family of methods which consist in adding an artificial diffusive term of order $h$ to the bilinear form of the continuous problem. Such a method improves the stability but not the convergence, and we expect to have uniform estimates of the same order as for the standard scheme. The simplest upwind technique of this kind is defined by adding $h\langle\nabla u, \nabla v\rangle$ to the bilinear form $a(\bullet, \bullet)$ (equation (2.1)); this procedure, unlike our schemes, ignores the local features of the flow described by the velocity $b$.

The contribution of an element $K$ to the artificial diffusion in (3.12) can be written as follows.

$$
\begin{aligned}
\frac{|K|}{3} \sum_{S \in \Sigma^{K}} \sum_{S^{\prime} \in \mathcal{N}^{K}(S)} \alpha_{S S^{\prime}}^{K} B_{S S^{\prime}}^{K}\left(\sigma_{S S^{\prime}}^{K} \cdot \nabla\right. & u\lceil K)\left(\sigma_{S S^{\prime}}^{K} \cdot \nabla v \mid K\right) \\
& =\frac{|K|}{3} \sum_{S \in \Sigma^{K}} \frac{B h}{2} \sum_{i, j=1}^{2} \Lambda_{i j}^{K}(S)\left(\frac { \partial u } { \partial x _ { i } } \lceil K ) \left(\frac{\partial v}{\partial x_{j}}\lceil K),\right.\right.
\end{aligned}
$$

where the matrix function $\Lambda^{K}$ is positive semidefinite and all its entries are bounded by

$$
\left|\Lambda^{K}(S)\right|<C h\left(S \in \Sigma^{K}\right),
$$

where $C$ is independent of $h, K, K$, and $S$, as in (3.14). Hence, we may look upon 
the bilinear form (3.15) as the result of numerical integration applied to

$$
a_{h}(u, v)=\kappa\langle\nabla u, \nabla v\rangle+\langle b \cdot \nabla u, v\rangle+c(u, v)+\frac{B h}{2} \sum_{i, j=1}^{2}\left\langle\Lambda_{i j} \frac{\partial u}{\partial x_{i}}, \frac{\partial v}{\partial x_{j}}\right\rangle .
$$

We shall prove hereafter convergence and stability for the solution of the discrete problem:

$$
\text { Find } u_{h} \in V_{0 h} \text { such that } a_{h}\left(u_{h}, v_{h}\right)=\left\langle f, v_{h}\right\rangle \quad \forall v_{h} \in V_{0 h} \text {. }
$$

Notice that existence and uniqueness are ensured, provided that $f \in \mathrm{L}^{2}(\Omega)$ and the matrix $\Lambda$ is positive semidefinite almost everywhere on $\Omega$. This last property implies also that the equation

$$
\|u\|_{\kappa, h}=a_{h}(u, u)^{\frac{1}{2}}
$$

defines a norm on $\mathrm{H}_{0}^{1}(\Omega)$.

Denoting by $\mathrm{W}^{1, \infty}(\Omega)$ the Sobolev space of functions which are, together with their first-order derivatives, in $\mathrm{L}^{\infty}(\Omega)$, we give some technical results in the next lemma.

Lemma 7.1 Assume that $c>0, \quad \kappa<\frac{1}{2} B h, \Lambda_{i j} \in \mathrm{W}^{1, \infty}(\Omega)(i, j=1,2)$, the $\mathrm{W}^{1, \infty}$ norm of these functions is bounded uniformly in $h$ and $\kappa$, and the matrix $\Lambda$ is symmetric positive semidefinite almost everywhere on $\Omega$. Then, one has

$$
\begin{gathered}
\|u\|_{0, \Omega} \leqslant C_{1}\|u\|_{\kappa, h} \quad \text { for } u \in \mathrm{H}_{0}^{1}(\Omega), \\
\|u\|_{\kappa, h} \leqslant C_{2}|u|_{1, \Omega} \text { for } u \in \mathrm{H}_{0}^{1}(\Omega), \\
\left|a_{h}(u, v)\right| \leqslant C_{3}|u|_{1, \Omega}\|v\|_{\kappa, h} \text { for } u, v \in \mathrm{H}_{0}^{1}(\Omega), \\
\left|a_{h}\left(u, w_{h}\right)-\left\langle f, w_{h}\right\rangle\right| \leqslant C_{4} h|u|_{2, \Omega}\left\|w_{h}\right\|_{h} \text { for } w_{h} \in V_{0 h},
\end{gathered}
$$

if $u \in \mathrm{H}^{2}(\Omega)$ is the solution of the continuous problem (2.2).

Proof. Properties (1) and (2) are direct consequences of Definition (7.5) and the Poincare inequality.

(3) From (7.3), one gets

$$
\left|a_{h}(u, v)\right| \leqslant|\langle\boldsymbol{b} \cdot \nabla u, v\rangle|+c|\langle u, v\rangle|+\left|\sum_{i, j=1}^{3}\left\langle\left(\kappa \delta_{i j}+\frac{B h}{2} \Lambda_{i j}\right) \frac{\partial u}{\partial x_{i}}, \frac{\partial v}{\partial x_{j}}\right\rangle\right| .
$$

The first two terms in the right-hand side are easily bounded by

$$
\tilde{C}_{3}|u|_{1}\|v\|_{0, \Omega} \leqslant \tilde{C}_{3} C_{1}|u|_{1}\|v\|_{\kappa, h},
$$

the latter inequality coming from Property (1). It remains to estimate the last term. Since the matrix with entries $a_{i j}=\kappa \delta_{i j}+\frac{1}{2} B h \Lambda_{i j}$ is positive definite, one can use the Cauchy-Schwarz inequality, which yields

$$
\left|\sum_{i, j=1}^{2}\left\langle a_{i j} \frac{\partial u}{\partial x_{i}}, \frac{\partial v}{\partial x_{j}}\right\rangle\right| \leqslant\|u\|_{\kappa, h}\|v\|_{\kappa, h} \leqslant C_{2}|u|_{1, \Omega}\|v\|_{\kappa, h} .
$$


(4) Equations (2.1), (2.2), (7.3), (7.4), and an integration by parts yield

$$
\begin{aligned}
\left|a_{h}\left(u, w_{h}\right)-\left\langle f, w_{h}\right\rangle\right| & =\frac{B h}{2}\left|\sum_{i, j=1}^{2}\left\langle\frac{\partial}{\partial x_{j}}\left(\Lambda_{i j} \frac{\partial u}{\partial x_{i}}\right), w_{h}\right\rangle\right| \\
& \leqslant C h|u|_{2, \Omega}\left\|w_{h}\right\|_{0, \Omega} \leqslant C C_{1} h|u|_{2, \Omega}\left\|w_{h}\right\|_{h} \quad\left(w_{h} \in V_{0 h}\right) .
\end{aligned}
$$

The convergence is stated in the following theorem.

THEOREM 7.2 If the solution $u$ of Problem (2.2) is in $\mathrm{H}^{2}(\Omega)$, with $f \in \mathrm{L}^{2}(\Omega)$, and $c>0$, and the hypotheses of Lemma 7.1 hold, then there exists a constant $C>0$, independent of $h$ and $\kappa$, such that

$$
\left\|u-u_{h}\right\|_{\kappa, h} \leqslant C h|u|_{2, \Omega},
$$

where $u_{h}$ is the solution of Problem (7.4).

Proof. Using Parts (2) and (3) of Lemma 7.1, instead of the continuity of the form $a_{h}(\bullet, \bullet)$ in the norm $\|\cdot\|_{\kappa, h}$, one can obtain, by a technique similar to the proof of the second Strang lemma,

$$
\left\|u-u_{h}\right\|_{\kappa, h} \leqslant C\left(\inf _{v_{h} \in V_{0 h}}\left|u-v_{h}\right|_{1, \Omega}+\sup _{w_{h} \in V_{0 h}} \frac{\left|a_{h}\left(u, w_{h}\right)-\left\langle f, w_{h}\right\rangle\right|}{\left\|w_{h}\right\|_{h}}\right) .
$$

The assertion follows by choosing $v_{h}$ as the interpolate of $u$ and applying Part (4) of Lemma 7.1.

Remarks. (1) Assume that the functions $\Lambda_{i j}$ are only in $\mathrm{L}^{\infty}(\Omega)$. Since the matrix $\Lambda$ is positive semidefinite, we may write

$$
\begin{aligned}
\frac{B h}{2} \mid \sum_{i, j=1}^{2}\left\langle\Lambda_{i j} \frac{\partial u}{\partial x_{i}}\right. & \left., \frac{\partial w_{h}}{\partial x_{j}}\right\rangle \mid \\
& \leqslant \frac{B}{2} h\left(\sum_{i, j=1}^{2}\left\langle\Lambda_{i j} \frac{\partial u}{\partial x_{i}}, \frac{\partial u}{\partial x_{j}}\right\rangle\right)^{\frac{1}{2}}\left(\sum_{i, j=1}^{2}\left\langle\Lambda_{i j} \frac{\partial w_{h}}{\partial x_{i}}, \frac{\partial w_{h}}{\partial x_{j}}\right\rangle\right)^{\frac{1}{2}} \\
& \leqslant h \kappa^{-\frac{1}{2}}|u|_{1, \Omega}\left\|w_{h}\right\|_{\kappa, h} .
\end{aligned}
$$

This means that we have, in place of (7.6),

$$
\left\|u-u_{h}\right\|_{\kappa, h} \leqslant h \kappa^{\frac{1}{2}}|u|_{2, \Omega} \text {. }
$$

Notice that the scheme defined by (3.12) satisfies the preceding assumption because the functions $\Lambda_{i j}$ can be obtained by linear interpolation of the nodal values on each triangle. To achieve the proof of the bound (7.7) for our conforming scheme, we have only to estimate the error due to numerical integration of the convective term. The technique is standard (see Ciarlet, $1978: \S 4.1)$ and requires a slightly modified version of the first Strang lemma. The key estimate is given by

$$
\begin{aligned}
\mid \int_{K} b_{j} \frac{\partial v_{h}}{\partial x_{j}} & w_{h} \mathrm{~d} x-\left.\sum_{S \in \Sigma^{K}} w_{h}(S) b_{j}(S)\left(\frac{\partial v_{h}}{\partial x_{j}} \mid K\right)\left|\leqslant C h^{2}\right|\left(\frac{\partial v_{h}}{\partial x_{j}} \mid K\right)|| w_{h}\right|_{1, K} \\
& \leqslant \bar{C} h\left|v_{h}\right|_{1, K}\left|w_{h}\right|_{1, K} \leqslant \bar{C}^{\prime} h K^{-\frac{1}{2}}\left|v_{h}\right|_{1, K}\left\|w_{h}\right\|_{K, h} \quad\left(v_{h}, w_{h} \in V_{0 h} ; j=1,2\right) .
\end{aligned}
$$


(2) For the nonconforming scheme defined by equations (5.17)-(5.18), we introduce, in place of (7.3), the form

$$
\begin{aligned}
a_{h}^{\prime}(u, v)= & \sum_{K \in \tau_{h}}\left(K\langle\nabla u, \nabla v\rangle_{K}+\frac{1}{2}\left(\langle\boldsymbol{b} \cdot \nabla u, v\rangle_{K}-\langle\boldsymbol{b} \cdot \nabla v, u\rangle_{K}\right)\right. \\
& \left.+\frac{B h}{2} \sum_{i, j=1}^{2}\left\langle\Lambda_{i j}^{K} \frac{\partial u}{\partial x_{i}}, \frac{\partial v}{\partial x_{j}}\right\rangle_{K}\right)+c\langle u, v\rangle,
\end{aligned}
$$

with the functions $\Lambda_{i j}^{K}$ defined from (5.17) by linear interpolation as indicated above. An estimate like (7.7), for $c>0, \kappa<\frac{1}{2} B h$, and a mesh $\tau_{h}$ belonging to a regular family of triangulations, is valid in this case too, in the norm

$$
\|u\|_{k, h}^{\prime}=a_{h}^{\prime}(u, u)^{\frac{1}{2}} \text {. }
$$

Actually, denoting by $u \in \mathrm{H}^{2}(\Omega)$ the solution of the continuous problem (2.2), integration by parts yields

$$
\begin{aligned}
\left|a_{h}^{\prime}\left(u, w_{h}\right)-\left\langle f, w_{h}\right\rangle\right| \leqslant K \mid \sum_{K \in \tau_{h}}^{2} \int_{\partial K} & \frac{\partial u}{\partial n} w_{h} \mathrm{~d} s\left|+\frac{1}{2}\right| \sum_{K \in \tau_{h}} \int_{\partial K} w_{h} u \boldsymbol{b} \cdot \boldsymbol{n} d s \mid \\
+ & \frac{B h}{2}\left|\sum_{K \in \tau_{h}}^{2} \sum_{i, j=1}\left\langle\Lambda_{i j}^{K} \frac{\partial u}{\partial x_{i}}, \frac{\partial w_{h}}{\partial x_{j}}\right\rangle_{K}\right| \quad\left(w_{h} \in V_{0 h}^{\prime}\right) .
\end{aligned}
$$

The first two absolute values in the right-hand side can be bounded, in a way analogous to the proof of Theorem 3 in Crouzeix \& Raviart (1973), by

$$
C h \kappa^{-\frac{1}{2}}|u|_{2, K}\left\|w_{h}\right\|_{\kappa, h},
$$

and similarly for the last term, since $\Lambda_{i j}^{K} \in \mathrm{L}^{\infty}(K)$. The numerical integration in (5.17), exact for polynomials of degree 2 , does not affect the convergence estimate.

(3) If the mesh contains equilateral triangles only, then the coefficients $\Lambda_{i j}$ corresponding to the scheme $\left(3.18^{\prime}\right)$ are in $\mathrm{W}^{1, \infty}(\Omega)$, and the conclusion of Theorem 7.2 holds.

\section{Numerical results and conclusion}

We have performed two tests in order to show the practical convergence of the schemes presented in this paper.

For the first test, the domain is the unit square $\Omega=(0,1) \times(0,1)$. We solved equation (1.1), with $b=(1,0), f=0, c=0, \quad \kappa=10^{-3}$, and the boundary conditions

$$
\begin{gathered}
u(0, y)=1 \quad \text { and } \quad u(1, y)=0 \quad(0 \leqslant y \leqslant 1) \\
\frac{\partial u}{\partial n}(x, 0)=\frac{\partial u}{\partial n}(x, 1)=0 \quad(0<x<1)
\end{gathered}
$$

The exact solution is given by

$$
u(x, y)=\left(\mathrm{e}^{x / \kappa}-\mathrm{e}^{1 / \kappa}\right) /\left(1-\mathrm{e}^{1 / \kappa}\right) \quad \text { for }(x, y) \in \bar{\Omega} .
$$




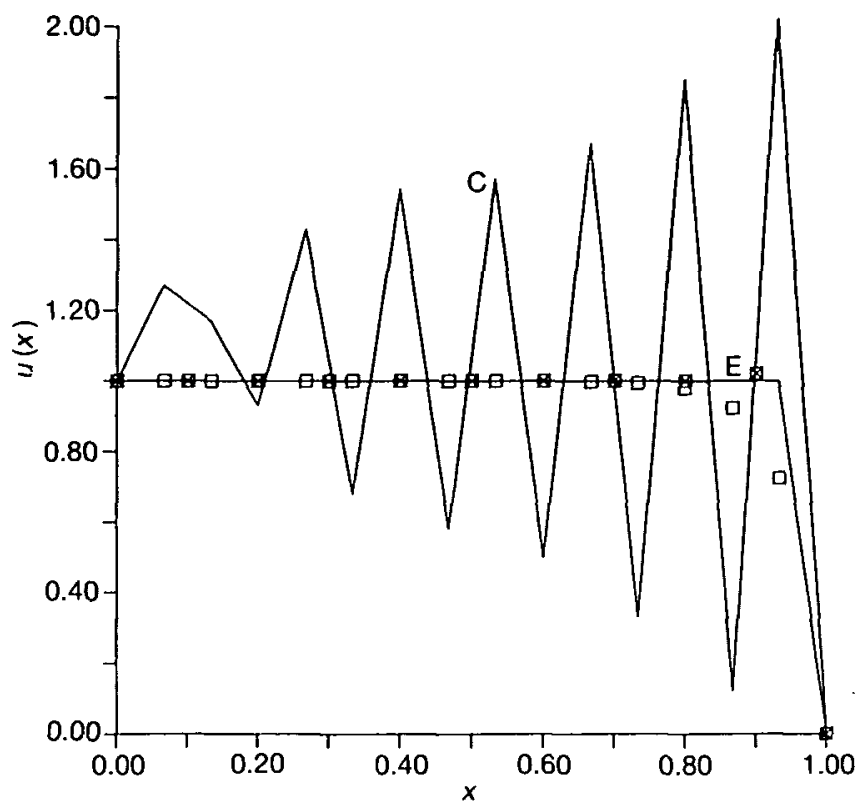

FIG. 1. Profiles of the solution along $y=\frac{1}{2}$. E: exact solution, C: centred scheme, $\square$ : conforming upwind scheme, $\bigotimes$ : nonconforming upwind scheme.

In Fig. 1, we present the profiles in the section $y=\frac{1}{2}$ of the exact solution, the standard conforming approximation, the upwind conforming approximation with the scheme (3.17), and the upwind nonconforming approximation with the scheme (5.20). The mesh consists of identical squares halved by parallel diagonals; we have 550 triangles with 256 nodes in the conforming case and 200 triangles with 320 nodes in the nonconforming one.

For the second test, the domain and its triangulation are given by Fig. 2. The velocity is $\boldsymbol{b}=(0,1)$, the external source is $f=1$ and $c=0$; the diffusion coefficient is $\kappa=10^{-3}$, and we impose the boundary condition $u\lceil\partial \Omega=0$. This problem is very difficult to solve, since there are boundary layers on $\partial \Omega$ at $x=0$, $x=2$, and $y=3$ and a transition layer at $x=1$ for $1<y<3$. Outside the layers, the exact solution can be approximated by the linear functions $u=y$ for $0<x<1$ and $u=y-1$ for $1<x<2$. The isolines for the upwind conforming schemes (3.17) and (3.18) can be found respectively in Fig. 3 and Fig. 4. As expected, the latter scheme is less diffusive than the former one.

Note that, for both tests, the upwind coefficients were calculated as if the triangulation were equilateral, by using the equals sign in inequalities (4.3) or (6.2).

The conforming and nonconforming upwind finite elements we have presented here satisfy, in a particular case, the monotonicity property. Results lacking uniform convergence, like those of Lemma 7.1, can be found in the literature (Ohmori \& Ushijima (1984), Ikeda (1983)). Except for a special case, we have only proved a uniform convergence of order $h \kappa^{-\frac{1}{2}}$ in the norm $\|\cdot\|_{\kappa, h}$ defined by 


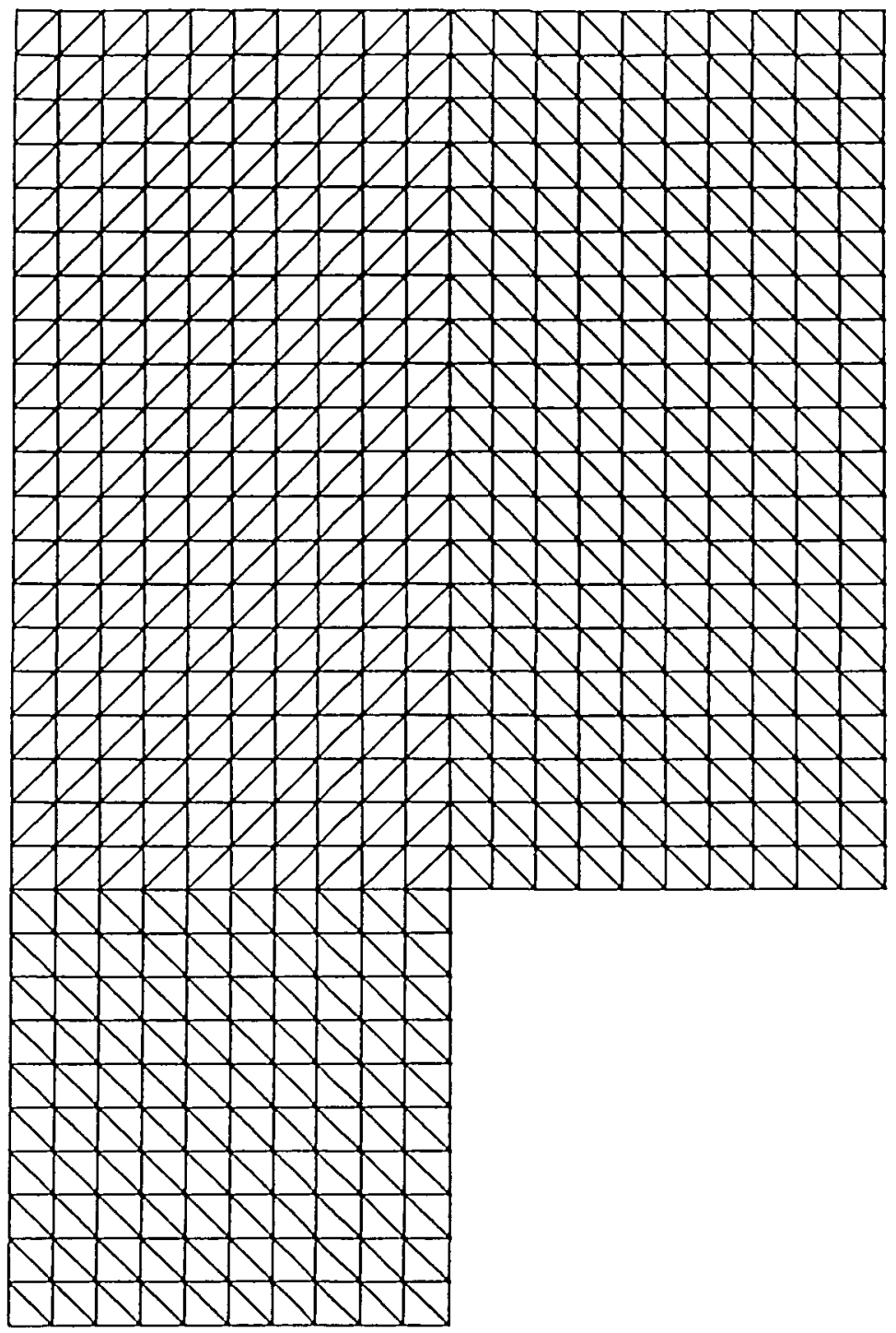

Fig. 2. The domain with its triangulation (1000 triangles, 551 nodes). The lower left corner has the coordinates $x=0, y=0$.

(7.3) and (7.5); this implies a convergence rate of order $h \kappa^{-1}$ in the norm $\|\bullet\|_{1, \Omega}$. Usually, convergence is discussed on the formulation without numerical integration (see Johnson et al., 1984), for which we have an $O(h)$ estimate for the conforming scheme in the $\|\bullet\|_{\kappa, h}$ norm; this means that the upwind process does not modify the consistency in this case. The generalization of these methods to 


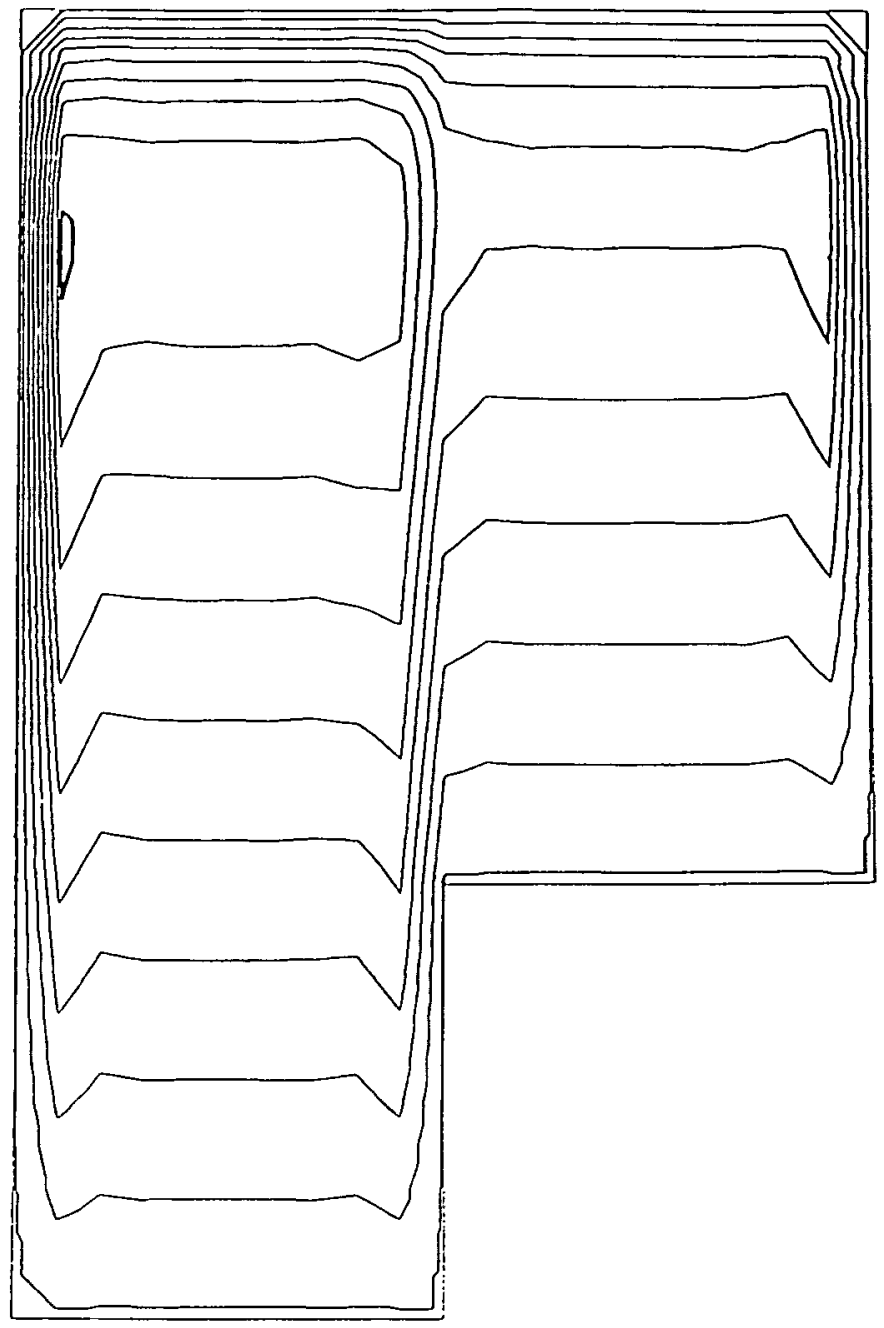

FIG. 3. Isolines of the solution for the upwind scheme (3.17). (15 equidistant isolines from 0 to 2.4).

the three-dimensional case and/or to incompressible Navier-Stokes equations is quite obvious.

\section{Acknowledgement}

The second author was supported by the Swiss National Science Foundation. 


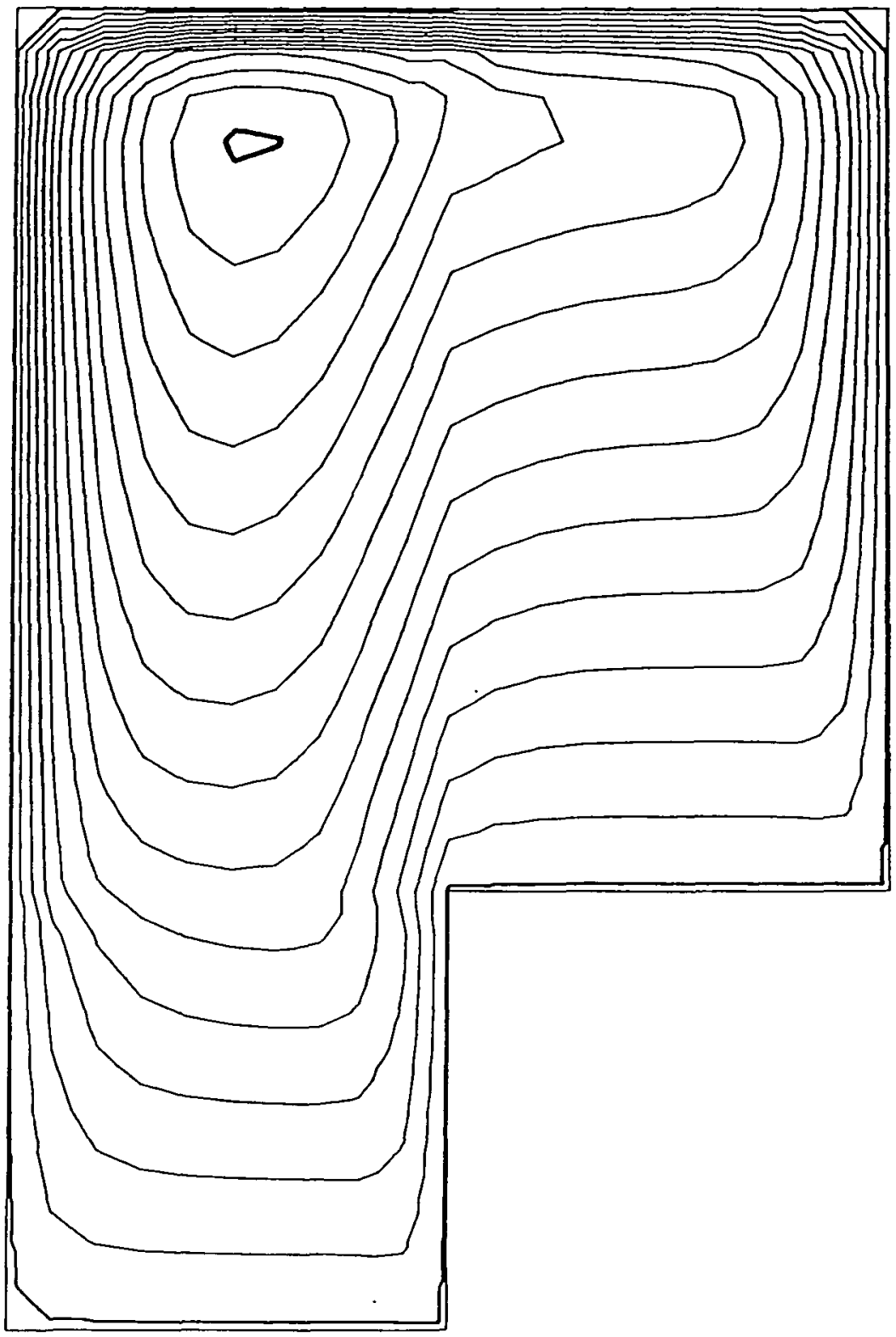

Fig. 4. Isolines of the solution for the upwind scheme (3.18). (Same values as Fig. 3.)

\section{References}

Berman, A., \& Plemmons, R. J. 1979 Nonnegative Matrices in the Mathematical Sciences, New-York: Academic Press.

Brooks, A. N., \& Hughes, T. J. R. 1982 Streamline-upwind Petrov-Galerkin formulations for convective dominant flows with particular emphasis on the incom- 
pressible Navier-Stokes equations. Comput. Methods Appl. Mech. Engng. 32, 199-259.

Ciarlet, P. G. 1978 The Finite Element Method for Elliptic Problems. Amsterdam: North-Holland.

Crouzeix, M., \& Raviart, P. A. (1973) Conforming and nonconforming finite element methods for solving the stationary Stokes equations I. RAIRO Anal. Numer. 3, 33-76.

Gilbarg, D., \& Trudinger, N. 1977 Elliptic Partial Differential Equations of Second Order. Grundlehren der mathematischen Wissenschaften 224. Berlin: Springer.

Hughes, J. T. R., Liu, W. K., \& Brooks, A. N. 1979 Review of finite element analysis of incompressible viscous flow by the penalty function formulation. J. Comp. Phys. 30, $1-40$.

IKEDA, T. 1983 Maximum Principle in Finite Element Models for Convection-Diffusion Phenomena. Lecture Notes in Numerical and Applied Analysis Vol. 4. Amsterdam: North-Holland.

Johnson, C., Navert, V., \& Pitkäranta, I. 1984 Finite element method for linear hyperbolic problems. Comput. Methods Appl. Mech. Engng. 45, 285-312.

Ohmori, K., \& UshiJima, T. 1984 A technique of upstream type applied to a linear conforming finite element approximation of convective diffusion equations. RAIRO Anal. Numer. 18, 309-332.

Richtmyer, R. D., and Morton, K. W. 1967 Difference Methods for Initial Value Problems. New York: Interscience.

Temam, R. 1984 Navier-Stokes Equations (revised edn) Amsterdam: North-Holland.

ThOMASSET, F. 1981 Implementation of Finite Element Methods for Navier-Stokes Equations. Berlin: Springer. 